\title{
Article
}

\section{Upper limb and eye movement coordination during reaching tasks in people with stroke}

Meadmore, KL, Exell, TA, Burridge, JH, Hughes, AM, Freeman, CT and Benson, Valerie

Available at http://clok.uclan.ac.uk/28041/

Meadmore, KL, Exell, TA, Burridge, JH, Hughes, AM, Freeman, CT and Benson, Valerie ORCID: 0000-0002-0351-4563 (2018) Upper limb and eye movement coordination during reaching tasks in people with stroke. Disability and rehabilitation, 40 (20). pp. 2424-2432. ISSN 0963-8288

It is advisable to refer to the publisher's version if you intend to cite from the work. http://dx.doi.org/10.1080/09638288.2017.1336649

For more information about UCLan's research in this area go to http://www.uclan.ac.uk/researchgroups/ and search for <name of research Group>.

For information about Research generally at UCLan please go to http://www.uclan.ac.uk/research/

All outputs in CLoK are protected by Intellectual Property Rights law, including Copyright law. Copyright, IPR and Moral Rights for the works on this site are retained by the individual authors and/or other copyright owners. Terms and conditions for use of this material are defined in the policies page.

\section{CLoK}

Central Lancashire online Knowledge www.clok.uclan.ac.uk

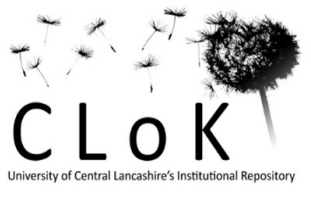


Upper Limb and Eye Movement Coordination during Reaching Tasks in People with Stroke.

Katie L. Meadmore ${ }^{1,2 *}$, Timothy A. Exell ${ }^{3,4}$, Jane H. Burridge ${ }^{2}$, Ann-Marie Hughes ${ }^{2}$, Christopher T Freeman ${ }^{4}$, Valerie Benson ${ }^{1}$.

${ }^{1}$ Psychology, Faculty of Social, Human and Mathematical Sciences, University of Southampton, Southampton, UK.

${ }^{2}$ Centre for Innovation and Leadership in Health Sciences, Faculty of Health Sciences, University of Southampton, Southampton, UK.

${ }^{3} \mathrm{~F}$ aculty of Science, University of Portsmouth, Portsmouth, UK

${ }^{4}$ Electronics and Computer Science, Faculty of Physical Sciences and Engineering, University of Southampton, Southampton, UK.

*corresponding author

Email: K.Meadmore@soton.ac.uk

Word count: 4671 


\begin{abstract}
Purpose: To enhance understanding of the relationship between upper limb and eye movements during reaching tasks in people with stroke.

Methods: Eye movements were recorded from ten control participants and eight chronic stroke participants during a visual orienting task (Experiment 1) and a series of reaching tasks (Experiment 2). Stroke participants completed the reaching tasks using (i) their less impaired upper limb, (ii) their more impaired upper limb without support, and (iii) their more impaired upper limb, with support (SaeboMAS gravitational support and/or electrical stimulation). Participants were tested individually and completed both experiments in the same session.

Results: Oculomotor control and the coordination between the upper limb and the oculomotor system were found to be intact in stroke participants when no limb movements were required, or when the less impaired upper limb was used. However, when the more impaired upper limb was used, success and accuracy in reaching decreased and patterns of eye movements changed, with an observed increase in eye movements to the limb itself. With upper limb support, patterns of hand-eye coordination were found to more closely resemble those of the control group.
\end{abstract}

Conclusion: Deficits in upper limb motor systems result in changes in patterns of eye movement behaviour during reaching tasks. These changes in eye movement behaviour can be modulated by providing upper limb support.

Key Words: Upper limb movement, Eye movements, Reaching Tasks, Stroke, Coordination, Rehabilitation. 


\section{Upper Limb and Eye Movement Coordination during Reaching Tasks in People with}

\section{Stroke.}

Knowledge regarding hand-eye coordination in neurologically impaired participants is sparse, and it is not clear what happens to hand-eye coordination when the eye or upper limb motor systems are impaired in some way, for example, through damage from stroke. The study reported here investigates this.

Visually guided hand movements have received a lot of attention with respect to the coordination between the eye and upper limb motor systems (handeye coordination). Typically a hand movement to a visually displayed target (whether it be pointing, reaching or grasping) tends to be accompanied by a saccadic eye movement to the target [e.g. 1-4]. Through these saccadic eye movements an integrated representation of the scene is built up, and, this, along with stored information in visual memory and proprioceptive information, is used to guide upper limb movements [e.g. 1-10]. Indeed, evidence suggests that impaired accuracy in upper limb movements occurs when eye movements do not follow normal patterns of fixation to key elements in the scene, or if the person is unable to fixate the target during the movement [e.g., 11-13].

Hand-eye coordination typically occurs in an ordered sequence of events, although there is some variability in the relative timings of these, depending on the task demands [e.g. 1-4]. For pointing or reaching tasks, eye movements tend to precede hand movements to the target. This is because the oculomotor system is biased to responding to sudden visual onsets [5]. The eye then typically remains fixated on the target until the movement task has been completed [e.g., the target 
has been reached by the hand; 6, 7]. This is referred to as gaze anchoring and facilitates a more accurate reaching movement [4].

Approximately $77 \%$ of stroke survivors are left with some degree of upper limb motor impairment [14]. Thus it is unsurprising that stroke research has shown that visually guided actions with the more impaired upper limb are slower, less accurate and less coordinated than those made with the less impaired upper limb or by control participants [15-17].

It is also estimated that about $70 \%$ of stroke survivors exhibit some sort of eye movement disorder post stroke. This includes difficulty in the ability to make fast eye movements, to move the eyes from one object to another, and to coordinate movements in both eyes [18-21]. These difficulties in eye movement control can ultimately result in difficulties in performing activities of daily living and poorer rehabilitation outcomes [18-20, 22, 23].

The aim of the present investigation was to examine the relationship between upper limb movements and eye movements during a simple reaching task in healthy control and chronic stroke participants. To meet the study aim, three main objectives were devised: (1) to establish whether there are any differences in oculomotor function between control and chronic stroke participants by measuring patterns of eye movements during a simple visual orienting task; (2) to characterize the relationship between upper limb movements and eye movements during a simple reaching task by recording eye movements and upper limb movements concurrently; (3) to examine whether facilitating upper limb movement by providing different levels of rehabilitative support to the upper limb would modulate the relationship between hand and eye co-ordination during the reaching task. 
Three hypotheses were generated in relation to each of these objectives. It was hypothesized that stroke participants would take longer to programme and hence initiate an eye movement to a target $\left(\mathrm{H}_{1}\right)$. It was anticipated that in addition to longer initiation times for eye movements and upper limb movements, the relationship between these movements would differ between control and stroke participants. Specifically, it was hypothesized that stroke participants might not demonstrate gaze anchoring due to their motor impairments $\left(\mathrm{H}_{2}\right)$. It was also hypothesized that any differences in the relationship between eye movements and upper limb movements would become less pronounced with increased upper limb support $\left(\mathrm{H}_{3}\right)$.

\section{Method}

\section{Study Design}

There were four phases to the study. (1) Fugl-Meyer Upper Limb assessment [24] to assess motor impairment in stroke participant's upper limb (see table 1); (2) Orienting eye tracking task (Experiment 1); (3) Simple reaching tasks (Experiment 2); (4) Visual and cognitive tasks to ensure that any differences in the experimental results were not due to differences in basic visual or cognitive ability (see table 1).

The study protocol was based on a novel design developed specifically for this study. The head mounted eye tracking system (Experiments 1 and 2) and the Microsoft kinect, SaeboMAS support and the ES control system (Experiment 2) have been used previously and are validated [25-29]

\section{Participants}


Ethical approval for the study was granted by the University of Southampton Psychology Ethics Board. All participants provided written informed consent. Twelve control participants were recruited from the University of Southampton Psychology Older Adult Volunteer Database and eight stroke participants (1.5-8 years post stroke) were recruited from the Faculty of Health Sciences Participant Database.

Stroke participants met the following inclusion criteria: (i) aged 18 years or older, (ii) able to provide written informed consent, (iii) have no significant neurological impairment (other than stroke), (iv) be medically stable, (v) diagnosed as having sustained a stroke at least 6 months prior to participating in the study, (vi) currently experience difficulty using their upper limb, (vii) demonstrate some volitional activity in the wrist and hand and (viii) have no contrindications for using electrical stimulation. Control participants met criteria i-iv outlined above and were also required to have no problems with the upper limb that caused pain or abnormal movement of the arm when extending the elbow. Two of the control participants were used as pilot participants and were excluded from later analyses due to protocol changes following their initial participation.

\section{Experiment 1: Oculomotor control.}

\section{Method}

This study used a between group (control vs stroke) experimental design to examine patterns of eye movements during a simple visual orienting task. Participants were required to fixate a visually displayed target when this appeared on a display in front of them (see Figure 1, Experiment 1). 
The experiment was custom programmed using a bespoke system that incorporated snowflake software (Nuiteq) to control the display, and a custom Matlab (R2012b) graphical user interface. Stimuli were presented on a vertically orientated, interactive, visual touch-screen (Promultis 10 touch 47" bespoke capacitive, PQ-Lab). A central fixation stimulus was maroon coloured. The eight target stimuli comprised a white cross inside a $10 \mathrm{~cm} \times 10 \mathrm{~cm}$ blue coloured square. The centre of each target stimulus was located $10 \mathrm{~cm}$ away from the middle of the central fixation point, positioned at angles of $0,45,90,135,180,225,270$ and 315 degrees (each target was equidistant from the central fixation; see figure 1).

For each experimental trial (see figure 1): The central fixation stimulus appeared in the middle of the screen for 3 seconds. This then disappeared with simultaneous presentation of a target for 1 second. After a 1 second blank screen the next trial began. There were 24 trials in the task; with a target appearing in each of the 8 locations three times. The targets were presented in the same fixed pseudo-random order to each participant. Participants were verbally instructed to look at the central fixation stimulus, and to move their eyes to the target stimulus as soon as it appeared and to remain looking there until the next trial.

Figure 1 about here

Eye movement data were recorded using a head mounted eye tracking system which incorporated a video-based infrared tracking system, consisting of two time-synchronized cameras (an eye camera and a scene camera) mounted onto a frame worn by the participant like a pair of goggles. The eye camera faced the 
participant's eye and recorded the movements made by the eye (including the pupil and corneal reflection). The scene camera recorded the scene in front of the participant as the task progressed. Both cameras were mounted over the right eye of the participant. Participants viewed the screen binocularly but only the movements of the right eye were recorded. The two video feeds were recorded using a Lenova Thinkpad, recording at $30 \mathrm{~Hz}$. Gaze points (i.e., where the eye was fixating) were identified using EyeCalibrator, an in-house software package developed at the University of Southampton.

To ensure constant viewing conditions, all participants used a chin rest and were seated $70 \mathrm{~cm}$ away from the touch screen monitor. The equipment could be worn over glasses, which meant that volunteers who needed glasses for normal vision could be included in the study. Before the task started, participants first completed a calibration task which required them to sequentially look at the centre of nine stimuli that were used for the task. The calibration procedure ensured that gaze locations across the display could be later computed for the eye movement recordings.

\section{Data preparation}

The eye movement data were inspected frame-by-frame and manually coded according to the region in which the participant's eyes landed. There were 10 regions of interest: the 8 target regions, the central fixation region, and the area outside these regions. In line with eye tracking methodology [25, 30-32] and to reduce variability in data, trials in which data were less than $60 \mathrm{~ms}$ or more than 3 
standard deviations from the group mean were excluded. This resulted in $4 \%$ of control participant data and $1 \%$ of stroke participant data being excluded.

\section{Outcome Measures}

Visual and Cognive tasks. For the stroke participants the history of the stroke was confirmed and the upper limb component of the Fugl-Meyer assessment [24] was completed to assess motor impairment. All participants completed a Snellen eye test to assess near visual acuity, the Montreal Cognitive Assessment [33] to assess cognitive impairment, the National Adult Reading Test [34] to assess verbal IQ, the Behavioural Inattention Test [35] to assess attentional deficits and the Visual Patterns Test [36] to assess short term visual memory.

Eye movements. Accuracy to target: number of trials (/24) in which participant's gazes landed on the target region after leaving the central region. This measure provided insight into the execution and programming accuracy of eye movements. Eye movement latency: time (in ms) for participant's to leave the central fixation region following the onset of a target. This measure provided insight into programming time and generation of eye movements.

Eye movement duration to reach target region: the average time (in ms) for participant's eye movements to reach the target region following it's onset. Note that this measure could comprise more than one eye movement, and in that case the eye movement durations were summed.

Average number of visits to a target region: the average number of times the participant's gaze landed on the target region following a gaze outside that region. 
This measure provided insight into the ability to maintain fixation in the target region.

\section{Results}

\section{Participants}

One of the stroke participants was excluded due to a deviation from protocol. Data are therefore reported for ten control and seven stroke participants. All 17 participants complied with the study protocol and no participants reported any adverse effects. All participants had normal or corrected-to-normal vision and none had visual neglect or visual field deficits. Both groups scored similarly on a battery of cognitive and visual tasks. Participant demographics are presented in table 1.

Table 1 about here

\section{Eye movement Data Analyses}

There were no significant group effects of any of the eye movement measures (Oneway ANOVA, see table 2). The ability to generate and execute eye movements to visual targets was intact and equivalent in both groups.

Table 2 about here

Experiment 2: Eye Movements and Upper Limb Movements during Reaching

\section{Method}


Experiment 2 used a mixed-participant design with Group (control vs stroke) as a between participant variable. Upper limb condition was a within-participant variable (see below for description). The set up was similar to Experiment 1. The task was custom programmed and also included use of a data capture card (dSPACE ds1103) for collection of upper limb movement data (detailed below) via a Microsoft Kinect ${ }^{\circledR}$ camera (Microsoft, Washington, USA). Figure 2 demonstrates the software flow for the hardware devices that were used during data collection.

Prior to each trial, the Microsoft Kinect ${ }^{\circledR}$ motion capture system was calibrated to ensure that it had identified the participant. Eye movements were calibrated prior to the start of the task. A chin rest was not used during the tasks so participants could move their head freely.

Figure 2 about here

The same stimuli that were used in Experiment 1 were also used in Experiment 2 (see figure 1). There were 16 different pre-determined movement trials which required participants to reach out with one arm and touch a visually displayed target. During half of the trials (static trials), the central fixation stimulus appeared for 3 seconds and was then replaced by a target located in one of the eight possible external positions. In the other half of trials (dynamic trials), following the central fixation stimulus, an initial target appeared briefly for 500 ms, before a new target simultaneously appeared in a different location (either 2 spaces anticlockwise or 2 spaces clockwise from the initial target). The target remained on screen until the 
participant had touched it or the time period (20 s) had elapsed. Figure 1 illustrates a static and a dynamic trial sequence.

The targets were presented in the same fixed pseudo-random order to each participant. Participants were seated at $80 \%$ of their arm length so that they could touch the screen with their index finger without moving their trunk from the back of the chair. Participants were verbally instructed to the look at the central fixation stimulus, and to move their arm to touch the center of the target stimulus as soon as it appeared. A self-selected rest period was provided between trials to prevent the effects of fatigue.

Control participants completed the task (i.e., 16 trials) using their dominant and non-dominant upper limb. Stroke participants completed the task in the following order: (i) using the less impaired upper limb (hereafter called unimpaired UL condition), (ii) using the more impaired upper limb (the impaired UL condition), (iii) using the more impaired upper limb with a SaeboMAS ${ }^{\circledR}$ arm support to deweight the arm (SaeboMAS condition) and (iv) using the more affected upper limb with both a SaeboMAS ${ }^{\circledR}$ arm support and electrical stimulation applied to the triceps and wrist extensors (ES condition).

For conditions (iii) and (iv), the participants more impaired arm was strapped into SaeboMAS ${ }^{\circledR}$, which still allowed the wrist to move freely. The researcher adjusted the support level to provide sufficient deweighting of the arm to facilitate movement in each participant individually. In the ES condition, stimulation was applied to both muscle groups as soon as each task started but the increase was graduated (i.e., did not go from 0 to maximum immediately) to ensure participant 
comfort $[26,28,29]$. Stroke participants only completed conditions (iii) and (iv) if they required more support.

\section{Data preparation}

Eye movement data

There were 10 regions of interest: the 8 target regions, the central fixation region, and a non-target region (which included other areas on the screen, and the participant's arm). Trials with poor calibration or in which eye movement latency data were less than $60 \mathrm{~ms}$ or more than $1000 \mathrm{~ms}$ were excluded. This resulted in $4 \%$ of trials being excluded for control data and $13 \%$ of trials being excluded for stroke participant data.

Limb Movement Data

Position data of the participants' wrists were low-pass filtered (Butterworth $4^{\text {th }}$ order) with cut-off frequencies determined using the autocorrelation method [37]. Filtered position data were then double-differentiated to calculate acceleration of the wrist, which was used to identify the commencement of arm movement (peak acceleration of wrist).

\section{Outcome Measures}

\section{Limb Movement}

Successful movement: defined as successfully touching the target on the screen within the display time period.

Time taken to initiate upper limb movement: the time taken for the upper limb to start moving following the onset of the target. 
Time taken to terminate the trial: the time taken from the target onset to when the target was touched. Note this measure was calculated for successful movements only.

Eye movements

Eye movement latency, Eye movement duration to reach the target region and Average number of visits to the target were the same as in Experiment 1. Note that for dynamic trials target refers to the second target that was presented, and the number of visits was summed across the two targets presented.

Average number of visits to non-target regions: the average number of times gaze landed on a region that was not the target, following onset of the target.

First Eye Movement to target: the proportion of trials where gaze went straight to the target after leaving the central region.

Eye Movement Initiated before Upper Limb Movement: the proportion of trials the eye movement was initiated before the upper limb movement.

\section{Participants}

Three control participants and one stroke participant were excluded due to poor calibration for the eye movement data. One control participant and one stroke participant were excluded due to poor calibration of upper limb movement. Data are therefore reported for six control and six stroke participants. All participants complied with the study protocol and no participants reported any adverse effects.

\section{Control Data}


A series of 2 (dominance: dominant vs non-dominant upper limbs) $\times 2$ (trial type: static vs dynamic) ANOVAs were conducted on all of the outcome measures. The pattern of data for dominance was very similar (only time to reach the target was significantly longer in a dynamic trial when using the non-dominant hand, $t s>2.85$, $p s<.036)$, and so data were collapsed across these two conditions to provide one combined control data set.

\section{Control data compared to data from the less impaired limb of stroke participants.}

The combined control data were compared to data from the unimpaired upper limb of the stroke participants. A series of 2 (trial type: static vs dynamic) $\times 2$ (participant group: control vs unimpaired UL) ANOVAS were conducted on the outcome measures. Table 3 displays these data.

Table 3 about here

There was a main effect of trial type for the time taken to initiate the upper limb movement, $F(1,10)=5.40, p=.043$, in the time taken to terminate the trial, $F(1,10)=19.03, p=.001$, in the eye movement duration to reach the target region, $F(1,8)=111.64, p=.000$, and in the number of visits made to the target, $F(1,10)=$ $2398.25, p=.000$. All participants took longer to initiate limb movements $(M=$ $1053.21 \mathrm{v} 1177.00 \mathrm{~ms})$ and to terminate the trial $(M=2261.15 \vee 2812.39 \mathrm{~ms})$ in the dynamic trials compared to the static trials. Participants also took longer to reach the target region with their eyes $(M=294.93 \vee 674.47)$, and made more visits to targets in the dynamic trials $(M=1.02 \vee 2.05)$, which reflects participants looking at both 
targets in the dynamic trials. There were no main effects of participant group and no interactions Fs $>4.00$, ps $<.07$.

\section{Comparisons of the more impaired upper limb under different support conditions}

Five stroke participants undertook the task using the SaeboMAS for support. One participant felt able to complete the task just as well with both arms (and scored 55/66 on the Fugl-Meyer assessment) and did not complete any further testing.

A series of 2 (trial type: static vs dynamic) $\times 3$ (condition: unimpaired UL vs impaired UL vs SaeboMAS) ANOVAS were conducted on the outcome measures. There was a main effect of condition for trial success, time taken for the upper limb movement to be initiated, and the number of visits to non-target regions ( $F s>5.08$, ps <.038). These results indicated that participants were more successful, initiated UL movement faster, and made fewer visits to non-target regions when they used their unimpaired arm compared to when using their more impaired arm or when they used their impaired UL with SaeboMAS support ( $t s>2.70, p s<.08$ ).

There was a main effect of trial type for eye movement duration to reach the target, $F(1,4)=28.83, p=.006$ and number of visits to $\mathrm{ROI}, F(1,4)=12.67, p=.024$. Participants made more visits to the target regions on dynamic than static trials $(\mathrm{M}=$ $2.28 \vee 2.93$ and $M=1.81 \vee 2.68$, respectively) and took longer to reach the target in dynamic compared to static trials (571.40 v 261.14).

A condition $x$ trial type interaction for trial success, $F(2,4)=5.71, p=.029$, showed that differences in trial success were driven by performance in the dynamic trials with participants being more successful when using the unimpaired arm 
compared to both when using their impaired arm without support and their impaired arm with SaeboMAS support, ts $>2.86, p \mathrm{~s}<.046$.

Table 4 about here

\section{Comparisons of the more impaired upper limb under ES conditions}

Three stroke participants also undertook the reaching tasks using the SaeboMAS and ES to facilitate their movement. One of these participants was excluded from the analysis due to poor calibration of the eye tracking and Microsoft Kinect systems.

Table 5 illustrates data from the two remaining participants. Descriptive analysis suggests that participants performance with the impaired arm became more like that observed for the unimpaired arm when participants were using support from the SaeboMAS and ES. The pattern of data also indicates that the addition of ES facilitated performance more than when only using SaeboMAS support.

Table 5 about here

\section{Discussion}

The main findings show that basic oculomotor function for generating and executing saccades to visually displayed targets in a simple orienting task was equivalent for healthy controls and chronic stroke participants. Patterns of timings for upper limb movements and eye movements were also very similar between control and stroke participants when performing reaching tasks with their less impaired upper limb. However, when using their more impaired upper limb, 
different patterns of eye movement behaviour were found in stroke participants, and the pattern of eye movements was affected by the amount of support the upper limb was receiving.

Experiment 1 established that the basic oculomotor system for generating and executing saccades to visually displayed targets was similar in control and chronic stroke participants; therefore, $\mathrm{H}_{1}$ (that oculomotor sampling would differ in these two groups) was rejected. For most trials, participants' gaze went directly to the target region following the target onset, and once their gaze landed on the target region of interest, they remained looking at that target (as instructed) until it disappeared. These findings of intact oculomotor control for simple visual orienting, as well as evidence of no other visual disturbances, suggest that eye movement deficits that are seen during acute stages of stroke may improve over time. However, formal confirmation of this remains to be tested empirically.

Experiment 2 investigated whether the relationship between patterns of eye movements and upper limb movements for reaching tasks differed following stroke. The findings showed a typical sequence of movements for this type of reaching task (see [6]): Following the onset of a visual target an eye movement was initiated and executed before the upper limb movement and, in general, gaze went straight to the target. Gaze then anchored to the target until the upper limb movement was complete. Thus, control and stroke participants (when using their less impaired upper limb) demonstrated gaze anchoring and accurate reaching in this task.

Both control and stroke participants were also able to successfully adapt their upper limb movements and eye gaze when the target position was changed in the dynamic trials. In addition, the finding that upper limb movements could be 
initiated without a direct fixation on the second target in the dynamic trials demonstrates the role of peripheral visual information in programming a motor movement. The data support the idea that the central nervous system can use an adaptive approach to coordinate eye and upper limb movements [9], and that, during reaching tasks, gaze anchoring and the visual feedback this provides is used to guide and update the planning and execution of ongoing upper limb movements [1, $7,11]$

The same pattern of findings was not replicated when stroke participants used their more impaired upper limb in the reaching task. Eye movements were again initiated before the upper limb movement and went straight to (or near to) the target; however, the eye did not remain stable on the target. Thus, gaze anchoring was not observed. Instead, eye gaze tended to switch between the computer screen and the upper limb. The finding of reduced gaze anchoring coupled with longer movement times reported for stroke particpants' impaired limbs, is in line with $\mathrm{H}_{2}$. Eye movements to the target have been shown to be more important than eye movements to the upper limb in reaching tasks [7, 38, 39]. In the current study it is likely that re-visits to the target were performed to monitor and update target location, providing visual feedback to the motor system to facilitate accurate reaching. Furthermore, in re-visits to non-target regions, the eye gaze often fell near to the target, suggesting that participants were using representations in short term memory to guide eye movements back to the target [9] (but these eye movements sometimes undershot the target location). This finding supports the notion that the observed re-visits to the target were for monitoring and updating purposes, and were not random patterns of eye movements. 
Monitoring and updating may also underlie the repeated eye movements made to the arm. Riddoch, Humphries and Bateman [40] suggested that given the close relationship between action and perception, visually fixating the upper limb whilst trying to initiate movement may facilitate patients in performing a motor action. Stroke participants may also have an impaired ability to extract proprioceptive information about the impaired limb when it is not in direct view. Alternatively, repeatedly looking to the limb may simply reflect frustration from the participant that planned motor commands are not being executed quickly or accurately. Further work is required to understand the reasons underlying the visual re-checking and the effects that these abnormal patterns of eye movements may have on performance.

Importantly, in line with $\mathrm{H}_{3}$, this study found that with support, upper limb performance and patterns of eye movements when using the more impaired upper limb became more like those observed in the control conditions in this study. Not surprisingly, performance was worst for stroke participants when using their more impaired upper limb alone, demonstrating the impact that stroke can have on simple motor tasks. In line with previous work [e.g., 28, 29, 41-44], gravitational support and electrical stimulation facilitated performance, with fewer eye movements made, shorter times to initiate the upper limb and better success rates in reaching to the targets. Indeed, these patterns were found in most outcome measures, and although the differences were not always statistically significant, this may reflect low power as a result of the small sample size and the increased variability often observed in this clinical population. It is noted that given these limitiations, the results of this study cannot be generalized to the wider population. 
In summary, oculomotor control for the generation and execution of saccades and the coordination between the oculomotor system and upper limb motor systems seems to be intact in chronic stroke participants when no limb movements are required or when the less impaired upper limb is used. However, when the more impaired upper limb is employed, the success in reaching decreases and the timing to complete the task, coupled with a propensity to visually recheck information increases. Importantly, however, more typical patterns of coordination re-emerge when using upper limb rehabilitative support. Thus, this novel experimental and interventional data set provides a solid foundation from which the relationship between the motor system for the upper limb and the oculomotor system has been highlighted. In conclusion, the findings from this study are the first to highlight how deficits in upper limb motor systems result in changes in patterns of eye movement behaviour during reaching tasks, and, that with upper limb support this impaired hand-eye coordination can return to patterns similar to those found for control data.

\section{Acknowledgements}

This study was funded by the Faculty of Human, Social and Mathematic Cciences, Strategic Interdisciplinary Research Development Fund. We would like to thank all the participants that took part in this study

\section{Declaration of Interest}

The authors report no conflicts of interest. The authors alone are responsible for the content and writing of this article. 
References

1. Gao KL, Ng SSM, Kwok JWY, et al. Eye-hand coordination and its relationship with sensorimotor impairments in stroke survivors. J Rehabil Med. 2010; 42: 368-373.

2. Land, M, Mennie N, Rusted, J. The roles of vision and eye movements in the control of activities of daily living. Perception. 1999; 28: 1311-1328.

3. Prado J, Clavagnier S, Otzenberger $\mathrm{H}$ et al. Two cortical systems for reaching in central and peripheral vision. Neuron. 2005; 48: 849-858.

4. Vercher JL, Magenes G, Prablanc, C et al. Eye-head-hand coordination in pointing at visual targets: spatial and temporal analysis. Exp Brain Res. 1994; 99 : p. 507-523.

5. Abrams RA, Meyer DA, Kornblum S. Eye-Hand Coordination: Oculomotor Control in Rapid Aimed Limb Movements. JEP: HPP. 1990; 16(2): 248-267.

6. Neggers SFW, Bekkering H. Ocular Gaze is Anchored to the Target of an Ongoing Pointing Movement. J Neurophysiol. 2000; 83(2): 639-651.

7. Rand MK, Stelmach GE. Effects of hand termination and accuracy on eye-hand coordination during sequential two-segment movements. Exp Brain Res. 2010; 207: 197-211.

8. Liversedge SP, Findlay JM. Saccadic eye movements and cognition. Trends Cogn Sci. 2000; 4: 6-14.

9. Issen LA, Knill DC. Decoupling eye and hand movement control: Visual shortterm memory influences reach planning more than saccade planning. J Vision. 2012; 12(1): 1-13.

10. Land MF. Eye movements and the control of actions in everyday life. Prog 
Retinal Eye Res. 2006; 25: 296-324.

11. Abrams, RA. Coordination of eye and hand for aimed limb movements. Vision Motor Contr. 1992; 129-152.

12. Crawford JD, Medendorp WP, Marotta JJ. Spatial transformations for eye-hand coordination. J Neurophysiol. 2004; 92:10-19.

13. Hesse C, Deubel H. Efficient grasping requires attentional resources. Vision Res. 2011;51:1223-1231.

14. Stroke Association. State of the nation: Stroke statistics. 2016. https://www.stroke.org.uk/sites/default/files/stroke_statistics_2015.pdf

15. Beer RF, Dewald JPA, Rymer WZ. Deficits in the coordination of multi-joint arm movements in patients with hemiparesis: evidence for disturbed control of limb dynamics. Exp Brain Res. 2000; 131: 305-319.

16. Levin MF. Interjoint coordination during pointing movements is disrupted in spastic hemiparesis. Brain 1996; 199: 281-293.

17. Zackowski KM, Dromerick AW, Sahrmann SA. How do strength, sensation, spasticity and joint individuation relate to the reaching deficits of people with chronic hemiparesis? Brain. 2004; 127: 1035-1046.

18. Pollock A, Hazelton C, Henderson CA, et al. Interventions for disorders of eye movement in patients with stroke. The Cochrane Library. 2011; 10: 1-33.

19. Rowe F, Brand D, Jackson CA, et al. Visual impairment following stroke: do stroke patients require vision assessment? Age Ageing. 2009; 38: 188-193.

20. Rowe F, Brand D, Jackson CA, et al. Reading difficulty after stroke: ocular and non ocular causes. Int J Stroke. 2011; 6: p. 404-411.

21. Stroke Association. Visual problems after strokes. 2012. Factsheet 37 (1). 
https://www.stroke.org.uk/sites/default/files/visual_problems_after_stroke.pdf

22. Jones $\mathrm{SA}$, \& Shinton RA. Improving outcome in stroke patients with visual problems. Age Ageing. 2006; 35:560-565.

23. Sand KM, Thomassen L, Melms A, et al. Visual impairment in stroke patients - a review. Acta Neurol Scand. 2013; 127: 52-56.

24. Fugl-Meyer A, Jääskö L, Leyman I, et al. The post-stroke hemiplegic patient. 1. A method for evaluation of physical performance. Scand J Rehabil Med. 1975; 7: 13-31.

25. Harland, B, et al. Modes of address in pictorial art: an eye movement study of Manet's Bar at the Folies-Bergère. Leonardo. 2014; 47(3): 241-247.

26. Sampson, P., Freeman, C.T., Coote, S., Demain, S., Feys, P., Meadmore, K. L. and Hughes, A. -M. Using functional electrical stimulation mediated by iterative learning control and robotics to improve arm movement for people with Multiple Sclerosis IEEE Transactions on Neural Systems and Rehabilitation Engineering. 2015; 24(2).

27. Kutlu, M., Freeman, C. T., Hallewell, E., Hughes, A.M. and Laila, D.S. Upper-Limb Stroke Rehabilitation using Electrode-Array Based Functional Electrical Stimulation with Sensing and Control Innovations. Medical Engineering \& Physics. 2016.

28. Meadmore KL, Hughes A-M, Freeman CT, et al. Functional Electrical Stimulation mediated by Iterative Learning Control and 3D robotics reduces motor impairment in chronic stroke. J NeuroEng Rehabil. 2012; 9(32): 1-11.

29. Meadmore KL, Exell T, Hallewell E, et al. The application of precisely controlled 
functional electrical stimulation to the shoulder, elbow and wrist for upper limb stroke rehabilitation: a feasibility study. J NeuroEng Rehabil. 2014; 11: 105-.

30. Howard, P, Liversedge, S \& Benson, V. Benchmark eye movement effects during natural reading in autism spectrum disorder. Journal of Experimental Psychology: Learning Memory and Cognition. 2016; 43: 109-127.

31. Shih, S, Meadmore, K and Liversedge, S. Aging, eye movements, and objectlocation memory. PLOS ONE. 2012; 7(3): e33485.

32. Benson V, letswaart M, Milner D. Eye Movements and Verbal Report in a Single Case of Visual Neglect. PLoS ONE. 2012; 7(8): e43743.

33. Nasreddine ZS, Phillips NA, Bédirian V, et al. The Montreal Cognitive Assessment, MoCA: A brief screening tool for mild cognitive impairment. J Amer Geriatrics Soc. 2005; 53: 695-99.

34. Nelson HE, Willison JR. The revised National Adult Reading Test: Test manual. Windsor: NFER-NELSON; 1991.

35. Wilson B, Cockburn J, Halligan P: Development of a behavioral test of visuospatal neglect. Arch Phys Med Rehabil. 1987; 68: 98-102.

36. Della Sala S, Gray C, Baddeley A, et al. The Visual Patterns Test: A new test of short-term visual recall. Suffolk, England: Thames Valley Test Company; 1997.

37. Challis JH. A Procedure for the Automatic Determination of Filter Cutoff Frequency for the Processing of Biomechanical Data. J Appl Biomech. 1999; 15(3): 303-317.

38. Biguer B, Prablanc C, Jeannerod M. The contribution of coordinated eye and head movements in hand pointing accuracy. Exp Brain Res. 1984; 55: 462-469. 39. Prablanc C, Pelisson D, \& Goodale MA. Visual control of reaching movements 
without vision of the limb: 1 . Role of retinal feedback of target position in guiding the hand. Exp Brain Res. 1986; 62: 293-302.

40. Riddoch MJ, Humphreys GW, Bateman A. Cognitive Deficits Following Stroke, Physiotherapy. 1995: 81: 465-473.

41. Krabben $\mathrm{T}$, Prange $\mathrm{GB}$, Molie $\mathrm{Bl}$, et al. Influence of gravity compensation training on synergistic movement patterns of the upper extremity after stroke, a pilot study. J NeuroEng Rehabil. 2012; 9: 44-55.

42. Prange GB, Jannink MJA, Stienen AHA, et al. An explorative, cross-sectional study into abnormal muscular coupling during reach in chronic stroke patients. J NeuroEng Rehabil. 2012; 7: 14-23.

43. Santos M, Zahmer LH, McKiernan et al. Neuromuscular electrical stimulation improves severe hand dysfunction for individuals with chronic stroke: A pilot study. J Neurol Phys Ther. 2006; 30: 175-183.

44. Thrasher TA, Zivanovic V, Mciiroy W, et al. Rehabilitation of reaching and grasping function $\mathrm{n}$ severe hemiplegic patients using functional electrical stimulation therapy. Neurorehabil Neural Re. 2008; 22: 706-714. 
Table 1.

Participant details for control and stroke groups.

\begin{tabular}{|c|c|c|c|}
\hline & $\begin{array}{l}\text { Control Group } \\
\text { Mean (Standar }\end{array}$ & $\begin{array}{l}\text { Stroke Group } \\
\text { Deviation), range }\end{array}$ & Results \\
\hline Side of lesion (L:R) & - & $2: 6$ & - \\
\hline Time since stroke & - & $4 y 5 m$ & - \\
\hline (years) & & $\begin{array}{l}(2 \mathrm{y} 5 \mathrm{~m}) \\
1.5-8 \text { years }\end{array}$ & \\
\hline Fugl-Meyer & - & 32.75 (15.00), & - \\
\hline Assessment (/66) & & $20-55$ & \\
\hline Age (years) & $\begin{array}{l}62(3.88), \\
55-69\end{array}$ & $\begin{array}{l}55.13(11.02) \\
42-72\end{array}$ & $F(1,17)=3.41, p=.08$ \\
\hline Gender (F:M) & $6: 4$ & $2: 6$ & $X=2.21, p=.18$ \\
\hline $\begin{array}{l}\text { Dominant/ "less } \\
\text { impaired" hand (R:L) }\end{array}$ & $8: 2$ & $6: 2$ & $X=.06, p=1.00$ \\
\hline Near visual acuity & $\begin{array}{l}78.4(60.29), \\
20-160\end{array}$ & $\begin{array}{l}85.5(62.52) \\
20-160\end{array}$ & $F(1,17)=.06, p=.81$ \\
\hline Years of education & $\begin{array}{l}16.1(2.08), \\
12-18\end{array}$ & $\begin{array}{l}15.25(2.82) \\
10-18\end{array}$ & $F(1,17)=.54, p=.47$ \\
\hline BIT star cancellation & .60 (1.07), & .50 (1.07), & $F(1,17)=.04, p=.85$ \\
\hline (error /54) & $0-3$ & $0-3$ & \\
\hline BIT letter cancellation & $1.9(2.85)$ & 1.25 (1.49), & $F(1,17)=.34, p=.57$ \\
\hline (errors /40) & $0-8$ & $0-4$ & \\
\hline
\end{tabular}




\begin{tabular}{llll}
\hline BIT line cancellation & $0(0)$ & $0(0)$ & - \\
$\begin{array}{l}\text { (errors /36 } \\
\text { NART verbal IQ }\end{array}$ & $9(5.77)$, & $14.50(11.59)$, & $F(1,16)=2.09, p=.17$ \\
(errors /50) & $2-18$ & $5-33$ & \\
MoCA (/30) & $28.11(1.36), 26-$ & $28.0(1.41)$, & $F(1,16)=.03, p=.87$ \\
& 30 & $26-30$ & \\
Visual Patterns Test & $8.82(1.58)$, & $8.80(1.58)$, & $F(1,17)=.001, p=.98$ \\
& $7-11.2$ & $7-11.4$ &
\end{tabular}

Note. $\mathrm{L}=$ Left; $\mathrm{R}=$ Right; $\mathrm{BIT}=$ Behavioural Inattention Test; NART = National Adult reading test; $\mathrm{MoCA}=$ Montreal Cognitive Assessment. 
Table 2

Eye movement measures for control and stroke groups and ANOVA results.

\begin{tabular}{|c|c|c|c|c|}
\hline & $\begin{array}{l}\text { Accuracy to } \\
\text { target } \\
\text { (proportion) }\end{array}$ & $\begin{array}{l}\text { Average of EM } \\
\text { latency (ms) }\end{array}$ & $\begin{array}{l}\text { Average of EM } \\
\text { duration to } \\
\text { reach target } \\
\text { region (ms) } \\
\text { D), range }\end{array}$ & $\begin{array}{l}\text { Average number } \\
\text { of visits to } \\
\text { region }\end{array}$ \\
\hline Control & .82 (.19), & 251.61 (26.97), & 267.39 (37.66), & $1(.01)$ \\
\hline Group & $.5-1$ & 210.38- 291.5 & $210.38-327.25$ & $1-1.04$ \\
\hline$N=10$ & & & & \\
\hline Stroke & .90 (.12) & 282.43 (53.90), & 301.77 (61.30), & $1(0)$ \\
\hline Group & $.65-1$ & $210.38-347.88$ & $213.13-365.75$ & $1-1$ \\
\hline$N=7$ & & & & \\
\hline ANOVA & $F(1,15)=.91$ & $F(1,15)=2.45, p$ & $F(1,15)=2.07, p$ & $F(1,15)=1.99, p$ \\
\hline & $p=.35$ & $=.14$ & $=.17$ & $=.18$ \\
\hline
\end{tabular}


Table 3.

Eye movement and UL movement outcome measures for the control and unimpaired limb conditions.

\begin{tabular}{|c|c|c|c|c|}
\hline & \multicolumn{2}{|c|}{ Control $(\mathrm{N}=6)$} & \multicolumn{2}{|c|}{ Unimpaired Limb $(\mathrm{N}=6)$} \\
\hline & Static & Dynamic & Static & Dynamic \\
\hline Successful & $.98(.05)$ & $1(0)$ & $.98(.05)$ & $1(0)$ \\
\hline \multicolumn{5}{|l|}{ movement } \\
\hline \multicolumn{5}{|l|}{ (proportion) } \\
\hline Time taken for UL to & 998.12 & 1171.11 & 1108.30 & 1182.89 \\
\hline be initiated (ms) * & $(249.97)$ & $(423.08)$ & $(162.96)$ & $(173.85)$ \\
\hline Time taken for & 2174.96 & 2726.07 & 2415.39 & 2819.76 \\
\hline movement to be & $(695.02)$ & $(695.28)$ & $(205.42)$ & (389.06) \\
\hline \multicolumn{5}{|l|}{ completed $(\mathrm{ms}) *$} \\
\hline Average number of & 1.03 & 2.07 & 1 & 2.03 \\
\hline visits to target * & $(.05)$ & $(.09)$ & (0) & (.08) \\
\hline Average number of & .03 & .05 & 0.02 & .14 \\
\hline visits to non-target & $(.05)$ & $(.08)$ & $(.05)$ & (.18) \\
\hline \multirow[t]{2}{*}{ EM latency (ms) } & 282.69 & 259.12 & 217.79 & 226.73 \\
\hline & (77.93) & $(42.96)$ & (52.89) & $(54.66)$ \\
\hline EM duration time to & 309.51 & 695.12 & 280.35 & 653.81 \\
\hline reach target $(\mathrm{ms}) *$ & $(84.54)$ & $(198.77)$ & $(52.11)$ & (94.22) \\
\hline EM before UL & .97 & .99 & 1 & 1 \\
\hline movement & $(.03)$ & $(.03)$ & & \\
\hline
\end{tabular}




\begin{tabular}{lllll}
\hline (proportion) & & & \\
First EM to target & .74 & .68 & .68 & .71 \\
(proportion) & $(.15)$ & $(.14)$ & $(.22)$ \\
\hline Note. $^{*}=$ significant differences in trial type; UL = upper limb; EM = eye movement
\end{tabular}


Table 4.

Eye movement and UL movement outcome measures for the unimpaired, impaired and SaeboMAS conditions.

\begin{tabular}{|c|c|c|c|}
\hline & $\begin{array}{l}\text { Unimpaired } \\
(\mathrm{N}=5)\end{array}$ & $\begin{array}{l}\text { Impaired } \\
(\mathrm{N}=5)\end{array}$ & $\begin{array}{l}\text { SaeboMAS } \\
(\mathrm{N}=5)\end{array}$ \\
\hline \multirow[t]{2}{*}{ Trial success (\%) \# } & 99 & 51 & 71 \\
\hline & $(.03)$ & $(.42)$ & $(.21)$ \\
\hline Time taken for the UL movement & 1180.21 & 1629.56 & 1628.40 \\
\hline to be initiated (ms) \# & $(109.55)$ & (159.33) & $(208.11)$ \\
\hline Time taken for trial to be & 2648.84 & 4256.43 & 5318.86 \\
\hline terminated (ms) & $(191.41)$ & $(2101.37)$ & $(720.87)$ \\
\hline Average number of visits to & 1.52 & 3.67 & 2.63 \\
\hline target * & $(.03)$ & (1.69) & $(1.33)$ \\
\hline Average number of visits to non- & 0.10 & 3.84 & 2.80 \\
\hline target regions \#* & $(.11)$ & $(2.77)$ & $(2.64)$ \\
\hline \multirow[t]{2}{*}{ EM latency } & 177.53 & 212.93 & 189.22 \\
\hline & $(85.36)$ & $(61.15)$ & $(71.86)$ \\
\hline EM duration time to reach target & 390.80 & 502.68 & 355.32 \\
\hline * & $(155.72)$ & $(242.77)$ & $(100.58)$ \\
\hline EM before UL movement & 1 & 1 & 1 \\
\hline \multirow[t]{2}{*}{ First EM to target } & .71 & .76 & .83 \\
\hline & $(.22)$ & $(.22)$ & $(.08)$ \\
\hline
\end{tabular}


Table 5.

Eye movement and upper limb movement outcome measures for the unimpaired, impaired SaeboMAS and ES conditions.

\begin{tabular}{|c|c|c|c|c|}
\hline & $\begin{array}{l}\text { Unimpaired } \\
(\mathrm{N}=2)\end{array}$ & $\begin{array}{l}\text { Impaired } \\
(\mathrm{N}=2)\end{array}$ & $\begin{array}{l}\text { SaeboMAS } \\
(\mathrm{N}=2)\end{array}$ & $\begin{array}{l}\text { ES } \\
(N=2)\end{array}$ \\
\hline \multirow[t]{2}{*}{ Trial success (\%) } & .97 & .34 & .77 & .87 \\
\hline & $(.05)$ & $(.06)$ & $(.24)$ & $(.10)$ \\
\hline Time taken for the UL movement & 1060.54 & 1559.83 & 1823.32 & 1511.34 \\
\hline to be initiated (ms) & $(38.63)$ & $(278.36)$ & $(322.44)$ & $(430.33)$ \\
\hline Time taken for trial to be & 2665.30 & 4700.00 & 4790.67 & 4662.19 \\
\hline terminated (ms) & $(454.15)$ & (954.59) & $(837.64)$ & $(38.65)$ \\
\hline Average number of visits to & 1.5 & 5.50 & 2.68 & 2.49 \\
\hline target & $(.00)$ & $(.53)$ & $(.75)$ & $(.40)$ \\
\hline Average number of visits to non- & .12 & 6.37 & 3.52 & 2.42 \\
\hline target & $(.17)$ & (.09) & $(2.09)$ & $(.88)$ \\
\hline \multirow[t]{2}{*}{ First EM to target } & .82 & .77 & .88 & .90 \\
\hline & $(.14)$ & (.09) & $(.00)$ & $(.15)$ \\
\hline
\end{tabular}

Note. $\mathrm{UL}=$ upper limb; $\mathrm{EM}$ = eye movement 